\title{
High expression of SLCO2B1 is associated with prostate cancer recurrence after radical prostatectomy
}

\author{
Tomoaki Terakawa ${ }^{1,2, *}$, Eriko Katsuta3,*, Li Yan ${ }^{4}$, Nitesh Turaga ${ }^{4}$, Kerry-Ann \\ McDonald $^{3}$, Masato Fujisawa ${ }^{2}$, Khurshid A. Guru ${ }^{1}$ and Kazuaki Takabe ${ }^{3,5,6,7,8}$ \\ ${ }^{1}$ Department of Urology, Roswell Park Cancer Institute, Buffalo, NY, USA \\ ${ }^{2}$ Department of Urology, Kobe University Graduate School of Medicine, Kobe, Japan \\ ${ }^{3}$ Department of Surgical Oncology, Roswell Park Cancer Institute, Buffalo, NY, USA \\ ${ }^{4}$ Department of Biostatistics and Bioinformatics, Roswell Park Cancer Institute, NY, USA \\ ${ }^{5}$ Department of Surgery, University at Buffalo, Jacobs School of Medicine and Biomedical Sciences, The State University of \\ New York Buffalo, NY, USA \\ ${ }^{6}$ Department of Breast Surgery and Oncology, Tokyo Medical University, Tokyo, Japan \\ ${ }^{7}$ Department of Surgery, Yokohama City University, Yokohama, Japan \\ ${ }^{8}$ Department of Surgery, Niigata University Graduate School of Medical and Dental Sciences, Niigata, Japan \\ *These authors contributed equally to this work \\ Correspondence to: Kazuaki Takabe, email: kazuaki.takabe@roswellpark.org \\ Keywords: prostate cancer; SLCO2B1; recurrence; EMT; OATP \\ Received: September 11,2017 Accepted: February 01,2018 Epub: February 08, 2018 Published: March 06, 2018 \\ Copyright: Terakawa et al. This is an open-access article distributed under the terms of the Creative Commons Attribution License \\ 3.0 (CC BY 3.0), which permits unrestricted use, distribution, and reproduction in any medium, provided the original author and \\ source are credited.
}

\section{ABSTRACT}

Solute carrier organic anion (SLCO) gene families encode organic anion transport proteins, which are transporters that up-take a number of substrates including androgens. Among them, high expression of SLCO2B1 is known to associate with the resistance to androgen deprivation therapy in prostate cancer $(\mathrm{PCa})$. We hypothesized that high expression of SLCO genes enhances PCa progression by promoting the influx of androgen. Here, we demonstrated the impact of the expression levels of SLCO2B1 on prognosis in localized PCa after radical prostatectomy (RP) utilizing 494 PCa cases in The Cancer Genome Atlas (TCGA). SLCO2B1 high expression group showed significantly worse Disease-free survival (DFS) after RP $(p=0.001)$. The expression level of SLCO2B1 was significantly higher in advanced characteristics including Gleason Score (GS $\leq 6$ vs GS $=7 ; p=0.047$, GS $=7$ vs GS $\geq 8 ; p=0.002$ ), pathological primary tumor (pT2 vs pT3/4; $<<0.001$ ), and surgical margin status (positive vs negative; $p=0.013$ ), respectively. There was a significant difference in DFS between these two groups only in GS $\geq 8$ patients $(p=0.006)$. Multivariate analysis demonstrated that only SLCO2B1 expression level was an independent predictor for DFS after RP in GS $\geq 8$. SLCO2B1 high expressed tumors in GS $\geq 8$ not only enriched epithelial mesenchymal transition (EMT) related gene set, $(p=0.027)$, as well as Hedgehog $(p<0.001)$, IL-6/JAK/STAT3 $(p<0.001)$, and K-ras signaling gene sets $(p<0.001)$, which are known to promote EMT, but also showed higher expression of EMT related genes, including $N$-cadherin $(p=0.024)$, SNAIL $(p=0.001)$, SLUG $(p=0.001)$, ZEB-1 $(p<0.001)$ and Vimentin $(p<0.001)$. In conclusion, PCa with high expression of SLCO2B1 demonstrated worse DFS, which might be due to accelerated EMT. 


\section{INTRODUCTION}

Prostate cancer $(\mathrm{PCa})$ is the most common malignant disease and the second leading cause of cancer related death among men in western industrialized countries [1]. Radical prostatectomy (RP) is the gold standard treatment for localized $\mathrm{PCa}$, however, approximately $30 \%$ of the cases develop recurrence after RP despite advances in surgical techniques [2]. Several pathological features such as positive surgical margins, extracapsular invasion, or seminal vesicle invasion were found to be associated with recurrence after RP [3, 4]. Pathological Gleason score (GS) has also been recognized as one of the most reliable prognostic factors predicting recurrence after RP [4-7].

To reduce the risk of recurrence, adjuvant treatment including radiotherapy or hormonal therapy has been suggested for patients with high risk pathological features $[3,8,9]$. However, the optimal adjuvant therapy for these high-risk patients is still a subject of continuous debate. Indeed, only $10-35 \%$ of the patients with extracapsular invasion develop recurrence [10-12] and the recurrence rate after RP differs immensely between studies [13]. These data suggest that current adjuvant therapies may include considerable overtreatment and indicate that a prognostic biomarker to precisely identify patients at highrisk of recurrence after RP is in urgent need.

Solute carrier organic anion (SLCO) gene families encode organic anion transport proteins (OATP), which are membrane transporters widely expressed in the human body that influx numerous compounds and drugs including androgens [14-16]. Several investigations have shown that SLCO genes, SLCO2B1 and SLCO1B3, mediate the uptake of androgen into PCa cells. SLCO2B1 transports one of the adrenal androgens, dehydroepiandrosterone sulfate (DHEAS), which is a precursor to the most potent androgen receptor into normal prostate and $\mathrm{PCa}$ cell. SLCO2B1 expression levels have been shown to correlate with DHEAS uptake in PCa cell lines [17]. On the other hand, SLCO1B3 transports testosterone, which is essential for progression of $\mathrm{PCa}[14,18]$. In agreement, the expression of these genes has been shown to be associated with the resistance to androgen deprivation therapy, which is the standard treatment for $\mathrm{PCa}[19,20]$. SLCO2B1 has been shown to be significantly increased in metastatic tissue, compared to primary tissue in $\mathrm{PCa}$ [21], and polymorphisms of SLCO2B1 gene is associated with shorter time to progression and overall survival (OS) in patients with metastatic PCa receiving androgen deprivation therapy $[17,22]$. These findings suggest that $\mathrm{SLCO} 2 \mathrm{~B} 1$ expression is associated with the progression of PCa.

However, the significance of SLCO2B1 gene expression on the recurrence after RP in $\mathrm{PCa}$ has not been investigated. We hypothesized that the expression level of SLCO genes will affect the progression of PCa and lead micro-metastasis by promoting the influx of androgen. In the present study, we investigated the impact of the expression level of SLCO2B1 on patient survival in localized PCa who underwent RP.

\section{RESULTS}

\section{The cancer genome atlas (TCGA) PCa patient cohort}

In whole TCGA PCa (PRAD) cohort, there were $494 \mathrm{PCa}$ cases with gene expression and survival data to analyze Disease-free survival (DFS) $(n=489)$ and OS $(n=494)$. The mean age of the cohort was $61.0 \pm$ 6.8 years old. The patient proportion of each pathologic primary tumor status for $\mathrm{pT} 2, \mathrm{pT} 3 \mathrm{a}, \mathrm{pT} 3 \mathrm{~b}$ and $\mathrm{pT} 4$ was 185 (37.4\%), 158 (32\%), 135 (27.3\%) and $10(20.2 \%)$, respectively (Table 1). DFS rates of 3, 5 and 10-year were $81.0 \%, 71.5 \%$ and $52.8 \%$, respectively (Figure $1 \mathrm{~A}$ ). OS rates of 3, 5 and 10-year were found to be $98.7 \%, 98.0 \%$ and $68.3 \%$, respectively (Figure 1B). According to GS of the prostatectomy specimens, $8.9 \%$ of patients had GS $\leq$ 6 , followed by $49.8 \%$ and $41.3 \%$ of patients with GS $=7$ and GS $\geq 8$, respectively (Table 1). Five-year DFS rates were $97.4 \%, 78.4 \%$ and $25.0 \%$ in $\mathrm{GS} \leq 6, \mathrm{GS}=7$ and $\mathrm{GS}$ $\geq 8$, respectively (Figure 1C). OS rates at 10-years were found to be $100 \%, 74.4 \%$ and $57.9 \%$ in the patients with $\mathrm{GS} \leq 6, \mathrm{GS}=7$ and $\mathrm{GS} \geq 8$, respectively (Figure 1D). These data demonstrate that patients in TCGA have more advanced disease compared to the recent reports from high volume institutions or multi-institutional studies since the proportion of pT2 patients is less than $40 \%$ in this cohort, whereas it surpassed $60 \%$ in recent reports [23-26]. The fact that patients with more advanced disease are included in this series leads the results of the higher proportion of high GS and inferior DFS comparing to recent reports.

\section{High expression of SLCO2B1, but not SLCO1B3, is associated with worse DFS after $\mathbf{R P}$ in $\mathbf{P C a}$}

First, we investigated the association of SLCO genes, SLCO2B1 and SLCO1B3, expression and prognosis of the $\mathrm{PCa}$ patients who underwent RP utilizing TCGA cohort. There are two patients $(0.4 \%)$ who have SLCO2B1 mutation and no patient has SLCO1B3 mutation, which suggests that there is minimal effect of mutation status on RNA expression. Patients were dichotomized into two groups, and the classification cutoff was determined by the mean value of the gene expression levels. Out of 494 cases, 192 and 302 patients were classified as SLCO2B1 high and low expression groups, respectively. We found that high expression of SLCO2B1 was associated with worse DFS after RP $(p=0.001)$, whereas there was no significant difference in OS between these two groups ( $p=0.837$ ) (Figure 2A, 2B). SLCO1B3 is another member of the SLCO gene family that influxes 
Patients $(n=494)$

\begin{tabular}{lc}
\hline Age (y.o.) $)^{\dagger}$ & $61.0 \pm 6.8$ \\
GS (\%) & $44(8.9 \%)$ \\
$\leq 6$ & $246(49.8 \%)$ \\
7 & $204(41.3 \%)$ \\
$\geq 8$ & $185(37.4 \%)$ \\
Pathological Primary tumor: pT (\%) & $158(32.0 \%)$ \\
pT2 & $135(27.3 \%)$ \\
pT3a & $10(20.2 \%)$ \\
pT3b & \\
pT4 & $343(69.4 \%)$ \\
Regional Lymph Node: $N(\%)$ & $79(16.0 \%)$ \\
N0 & \\
N1 & $313(63.4 \%)$ \\
Surgical margin status (\%) & $152(30.8 \%)$ \\
negative & \\
positive & $246(49.8 \%)$ \\
Adjuvant radiation therapy $(\%)$ & $40(8.1 \%)$ \\
no & \\
yes &
\end{tabular}

†: mean \pm SD
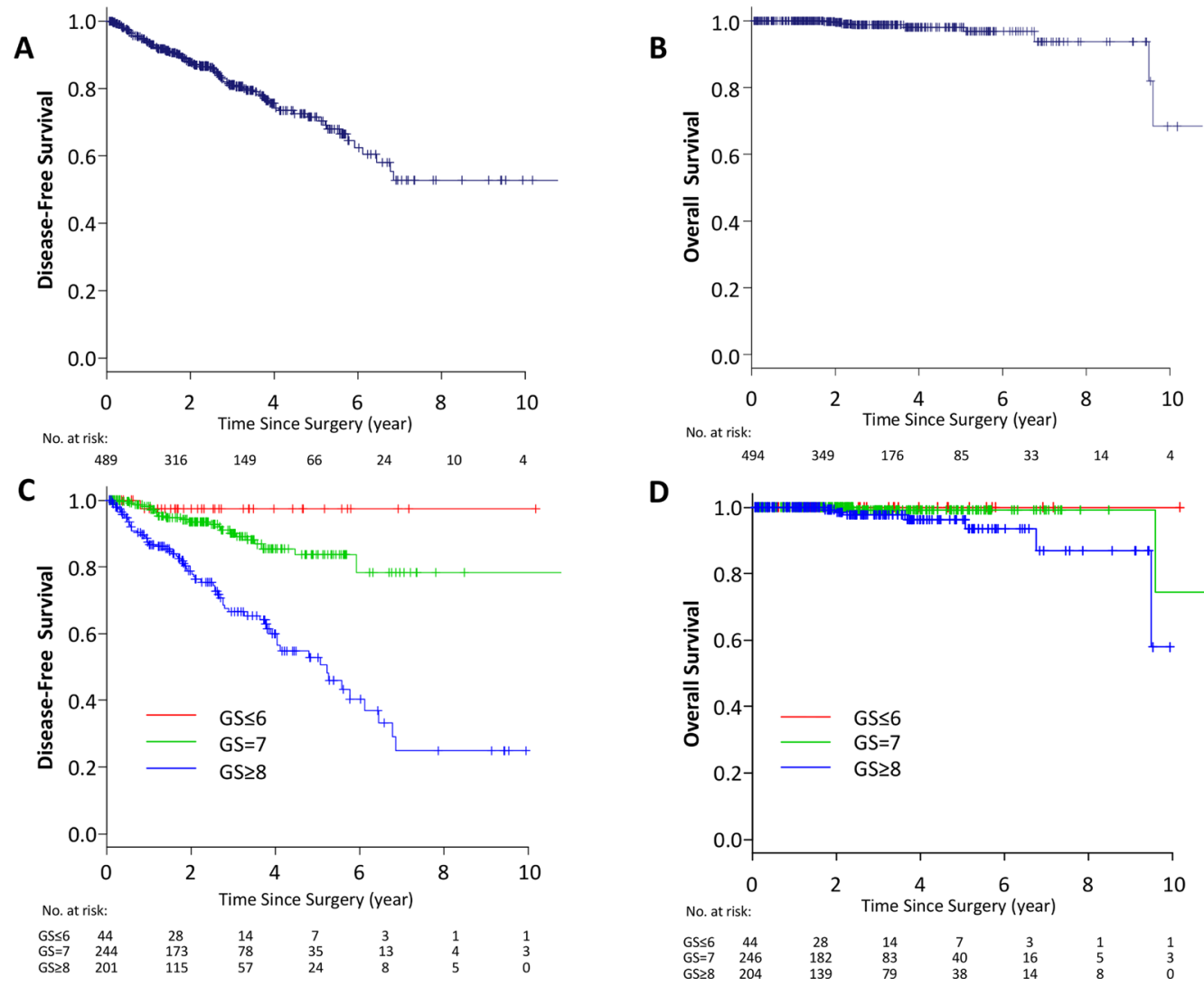

Figure 1: Kaplan-Meier estimate of survival in TCGA PCa patients. DFS (A) and OS (B) in whole TCGA PCa patients. DFS (C) and OS (D) classified by GS in whole TCGA PCa patients. Red line; GS $\leq 6$, green line; GS = 7, blue line GS $\geq 8$. 
androgens into PCa cells. In contrast to SLCO2B1, there was no significant difference in OS or DFS between high and low expression levels of SLCO1B3 (Figure 2C, 2D). The findings that high SLCO2B1 expressing tumors have worse DFS but no difference in OS were validated using the median cutoff in TCGA dataset (Supplementary Figure 1A, 1B).

\section{High SLCO2B1 expression is associated with aggressive pathological features}

SLCO2B1 expression levels were analyzed in different GS, pT status and surgical margin status. GS is one of the most reliable pathological parameters to determine aggressiveness of PCa, and positive surgical margins suggest the aggressive biology of a tumor. Interestingly, SLCO2B1 expression levels were all equivocally elevated in more advanced histological features such as GS (GS $\leq 6$ vs GS $=7 ; p=0.047, \mathrm{GS}=7$ vs $\mathrm{GS} \geq 8 ; p=0.002), \mathrm{pT}(\mathrm{pT} 2$ vs $\mathrm{pT} 3 / 4 ; p<0.001)$, and surgical margin status (negative vs positive; $p=0.013$ ) (Figure 3).

\section{A}

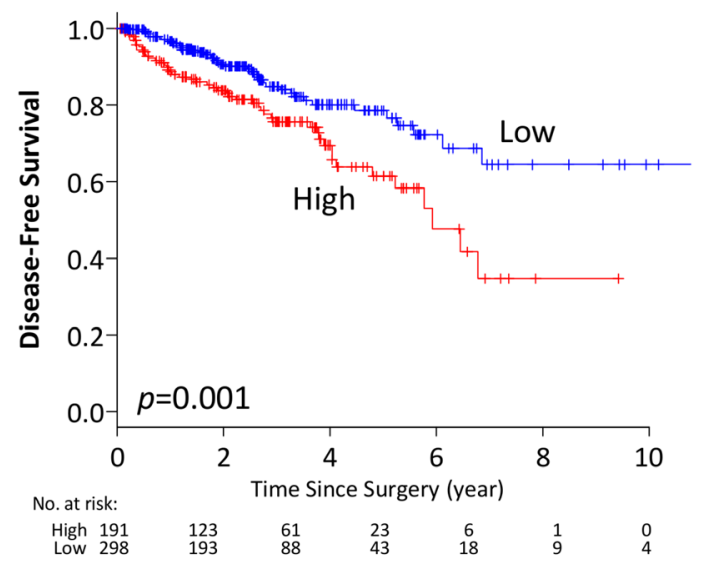

\section{C}

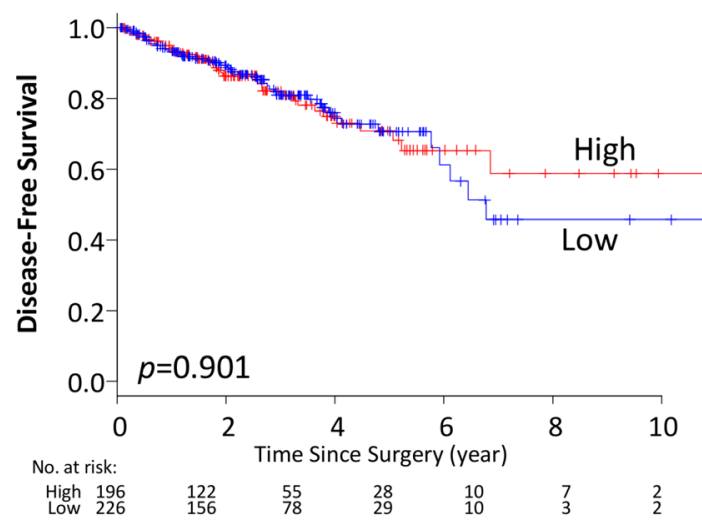

High expression of SLCO2B1 is a prognostic biomarker in GS high, but not in GS low tumors

Since SLCO2B1 expression was significantly higher in GS high tumors, we hypothesized that SLCO2B1 expression was associated with tumor aggressiveness in GS high tumors. Strikingly, high expression of SLCO2B1 was significantly associated with worse DFS only in GS $\geq$ 8 population $(p=0.006)$, and not in the other GS (GS $\leq 6$; $p=0.640, \mathrm{GS}=7 ; p=0.653$ ) (Figure 4). These findings indicate that SLCO2B1 expression could be a prognostic biomarker of recurrence after $\mathrm{RP}$ in $\mathrm{GS} \geq 8$ patients. The findings that high SLCO2B1 expressing tumors have worse DFS in GS $\geq 8$ but not in GS $\leq 7$ patients were validated using the median cutoff in TCGA dataset (Supplementary Figure 1C-1E).

\section{Clinicopathological features of patients with SLCO2B1 high expression among GS $\geq 8$ tumors}

Since high expression of SLCO2B1 associated with cancer recurrence only in GS $\geq 8$ patients,
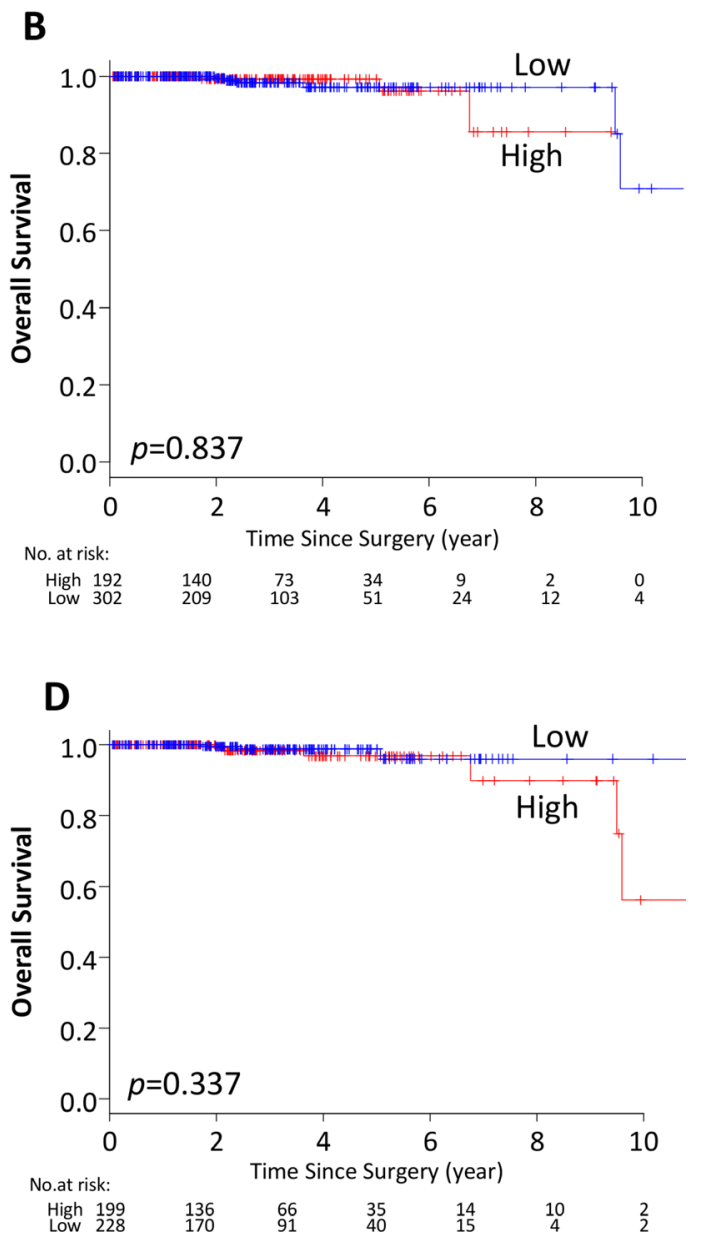

Figure 2: Kaplan-Meier estimate of disease-free and overall survival by dichotomized of SLCO2B1 and SLCO1B3 expression in TCGA PCa patients. (A, B) Classified by SLCO2B1 expression level, (C, D) classified by SLCO1B3 expression level. Red line; high expression, blue line; low expression of each genes. 
clinicopathological features were compared between SLCO2B1 high and low expression groups in PCa patients with GS $\geq 8$ to investigate whether certain populations associated with SLCO2B1 expression (Table 2). Interestingly, there was no significant difference in age, pT status (pT2 vs pT3/4), lymph node metastasis, surgical margin status, and postoperative radiation therapy between these two groups.

\section{SLCO2B1 is the only independent predictor for DFS in the patients with GS $\geq 8$}

We investigated the influence of SLCO2B1 expression in DFS compared to other clinical or pathological factors known to impact PCa recurrence by univariate and multivariate analyses using Cox proportional hazards regression. Pathological primary
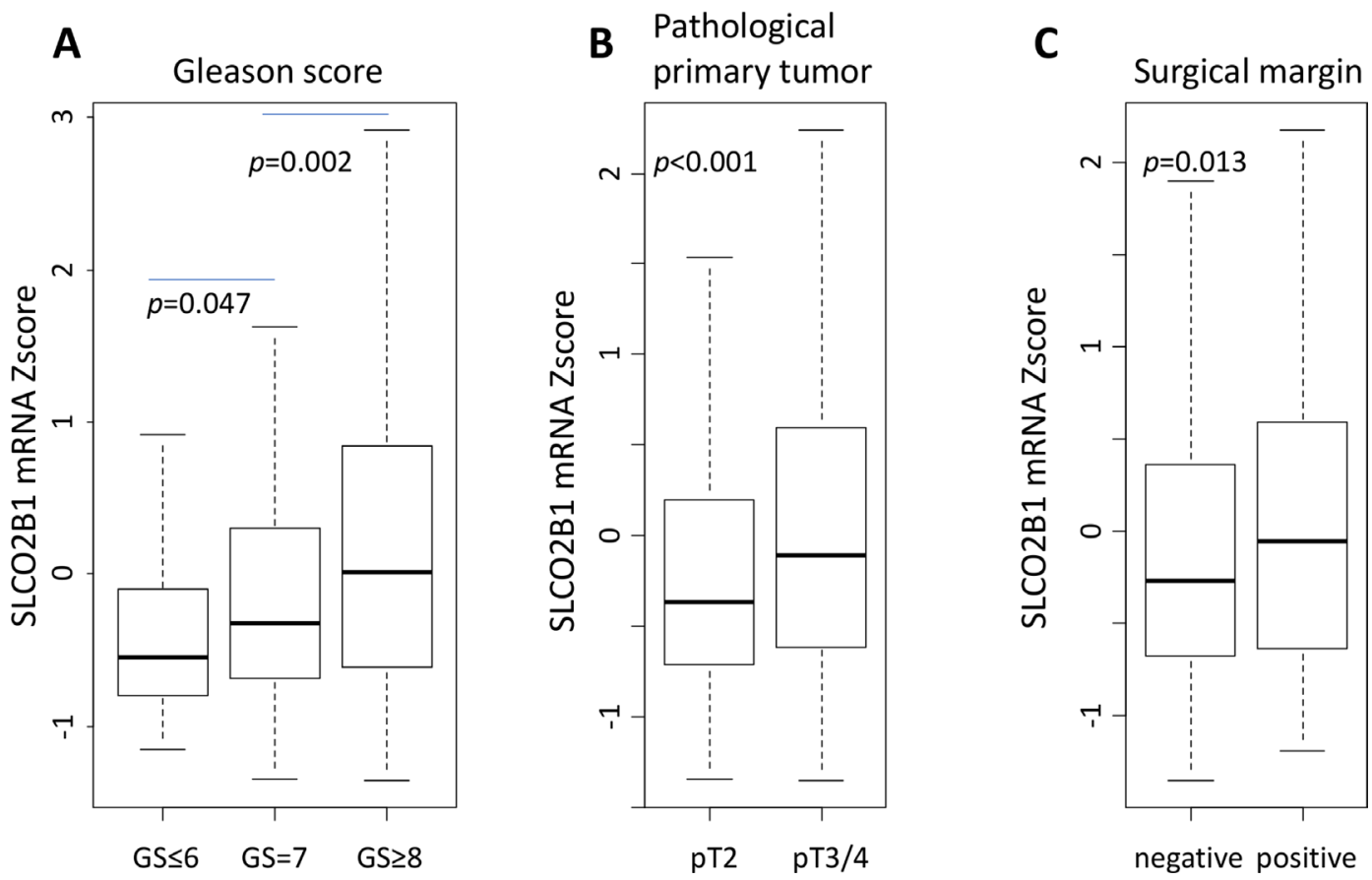

Figure 3: The expression level of SLCO2B1 according to pathological factors in TCGA PCa patients. (A) GS. (B) pathological primary tumor status (pT). (C) surgical margin status.

A

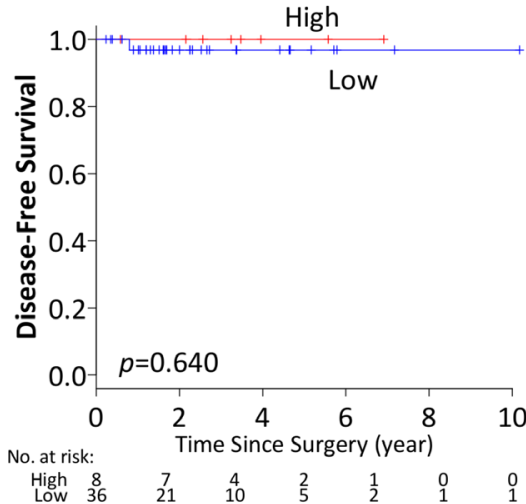

B

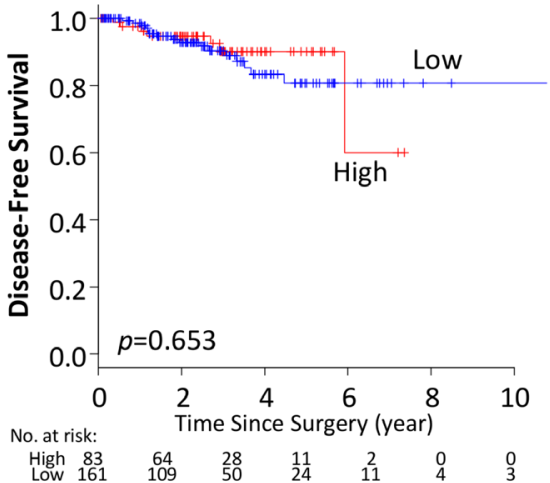

C

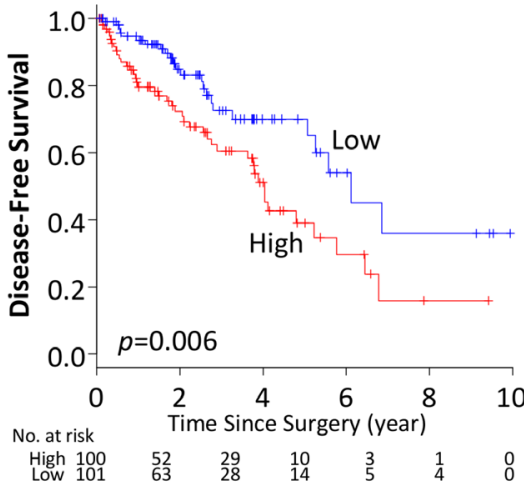

\section{$\mathrm{GS} \leq 6$}

\section{$\mathrm{GS}=7$}

\section{$\mathrm{GS} \geq 8$}

Figure 4: DFS classified by SLCO2B1 expression level in the subgroups according to GS in TCGA PCa patients. (A) $\mathrm{GS} \leq 6,(\mathbf{B}) \mathrm{GS}=7,(\mathbf{C}) \mathrm{GS} \geq 8$. Red line; high expression, blue line; low expression of SLCO2B1. 
Table 2: Clinicopathological characteristics of SLCO2B1 high and low in the PCa patients with GS $\geq 8$

\begin{tabular}{lccc}
\hline & $\begin{array}{c}\text { High } \\
(\boldsymbol{n}=\mathbf{1 0 1})\end{array}$ & $\begin{array}{c}\text { Low } \\
(\boldsymbol{n}=\mathbf{1 0 3})\end{array}$ & $\boldsymbol{p}$ \\
\hline Age (y.o.) & $62.2 \pm 5.6$ & $62.6 \pm 7.3$ & 0.666 \\
Pathological Primary tumor: pT & & & 0.309 \\
pT2 & 11 & 17 & \\
pT3a & 25 & 37 & 0.648 \\
pT3b & 60 & 45 & \\
pT4 & 5 & 2 & 0.564 \\
Regional Lymph Node: N & & & \\
N0 & 64 & 63 & \\
N1 & 30 & 35 & 0.570 \\
Surgical Margin & & 48 & \\
negative & 45 & 46 & \\
positive & 53 & 61 & \\
Adjuvant RT & & 18 & \\
no & 50 & & \\
yes & 19 & & \\
\hline
\end{tabular}

: mean \pm SD.

tumor status $(p=0.048)$, surgical margin status $(p=0.030)$ and SLCO2B1 expression $(p=0.007)$ were significant prognostic factors in the univariate analysis. Interestingly, multivariate analysis revealed that high expression of SCLO2B1 was the only independent prognostic factor for DFS $(p=0.022)$ (Table 3$)$.

\section{Gene expression between high and low expression of SLCO2B1 in PCa with GS $\geq 8$}

To investigate the mechanism of how high expression of SLCO2B1 is associated with high recurrence rate in the patients with $\mathrm{GS} \geq 8$ after $\mathrm{RP}$, Gene Set Enrichment Analysis (GSEA) was conducted. GSEA demonstrated that numerous genes were differently expressed between high and low expression of SLCO2B1. 2908 genes were upregulated with score $>3.0$ of GSEA, and 1411 genes were downregulated in the SLCO2B1 high expression group with score $<-3.0$ of GSEA. The heatmap of the top 50 upregulated and downregulated genes are shown in Supplementary Figure 2, as well as the list of genes (Supplementary Table 1).

\section{Key cancer signaling pathways related gene sets were enriched in SLCO2B1 high with GS $\geq 8$}

GSEA demonstrated that among 50 hallmark gene sets, 11 gene sets were significantly enriched in SLCO2B1 high expressed tumors with family wise error rate (FWER) $p<0.05$ (Supplementary Table 2), and 1 gene set was significantly enriched in SLCO2B1 low with FWER $p<$ 0.05 (Supplementary Table 3). Of those, SLCO2B1 high expressed tumors enriched a gene set related to epithelial mesenchymal transition (EMT), which is known to be one of the major mechanisms of metastasis and recurrence (Normalized enrichment score; NES $=2.02$, FWER $p=$ $0.027)$, as well as some of the pathway related gene sets known to promote EMT [27-30], including Hedgehog signaling gene set (NES $=2.19$, FWER $p<0.001)$, IL-6/ JAK/STAT3 signaling gene set $(\mathrm{NES}=2.21$, FWER $p<$ $0.001)$ and K-ras signaling gene set (NES $=2.29$, FWER $p<0.001$ ) (Figure 5). These gene sets were also enriched in SLCO2B1 high expressed tumors in GS $\leq 7$ tumors (Supplementary Figure 3).

\section{High expression of SLCO2B1 significantly upregulated EMT related gene expression in PCa with GS $\geq 8$}

In agreement with the GSEA results, the expression level of some EMT related genes, including N-cadherin ( $p$ $=0.024)$, SNAIL $(p=0.001)$ and SLUG $(p=0.001)$, ZEB-1 $(p<0.001)$ and Vimentin $(p<0.001)$, were significantly higher in SLCO2B1 high group in GS $\geq 8$ (Figure 6). This result is in sync with our previous publication that EMT is strongly associated with recurrence after RP [31, 32]. Therefore, one of the mechanisms responsible for the worse prognosis of SLCO2B1 high expression after RP may be due to promoted EMT.

\section{DISCUSSION}

In this study, we found that high expression of SLCO2B1, which mediates the uptake of DHEAS into 
Table 3: Univariate and multivariate analyses for independent DFS predictor of PCa patients with GS $\geq 8$

\begin{tabular}{|c|c|c|c|c|c|c|}
\hline \multirow[b]{2}{*}{ Clinicopathological factor } & \multicolumn{3}{|c|}{ Univariate analysis } & \multicolumn{3}{|c|}{ Multivariate analysis } \\
\hline & HR & $(95 \% \mathrm{CI})$ & $p$ & HR & $(95 \% \mathrm{CI})$ & $p$ \\
\hline Age $(>70$ vs $<70)$ & 0.884 & $(0.352-2.217)$ & 0.792 & & & \\
\hline $\mathrm{pT}(\mathrm{pT} 3,4$ vs pT2) & 2.518 & $(1.007-6.299)$ & $0.048^{*}$ & 2.223 & $(0.867-5.679)$ & 0.096 \\
\hline $\mathrm{N}$ (N1 vs N0) & 0.852 & $(0.495-1.468)$ & 0.564 & & & \\
\hline $\begin{array}{l}\text { Surgical margin (positive vs } \\
\text { negative) }\end{array}$ & 1.750 & $(1.057-2.896)$ & $0.030^{*}$ & 1.607 & $(0.96-2.692)$ & 0.071 \\
\hline $\begin{array}{l}\text { Adjuvant Radiation (yes } \\
\text { vs no) }\end{array}$ & 1.038 & $(0.55-1.959)$ & 0.909 & & & \\
\hline SLCO2B1 (High vs Low) & 1.991 & $(1.204-3.294)$ & $0.007^{*}$ & 1.829 & $(1.093-3.061)$ & $0.022^{*}$ \\
\hline
\end{tabular}

${ }^{*}: p<0.05$.

$\mathrm{PCa}$ cells, is associated with advanced pathological features in PCa including GS as well as worse DFS after RP particularly among high GS patients. Furthermore, we elucidated that high SLCO2B1 expression tumors enriched EMT related gene sets as well as key cancer signaling pathways related gene sets that promote EMT. These results imply that this may be one of the mechanisms responsible for the worse prognosis after RP in SLCO2B1 high expressed tumor. To our knowledge, this is the
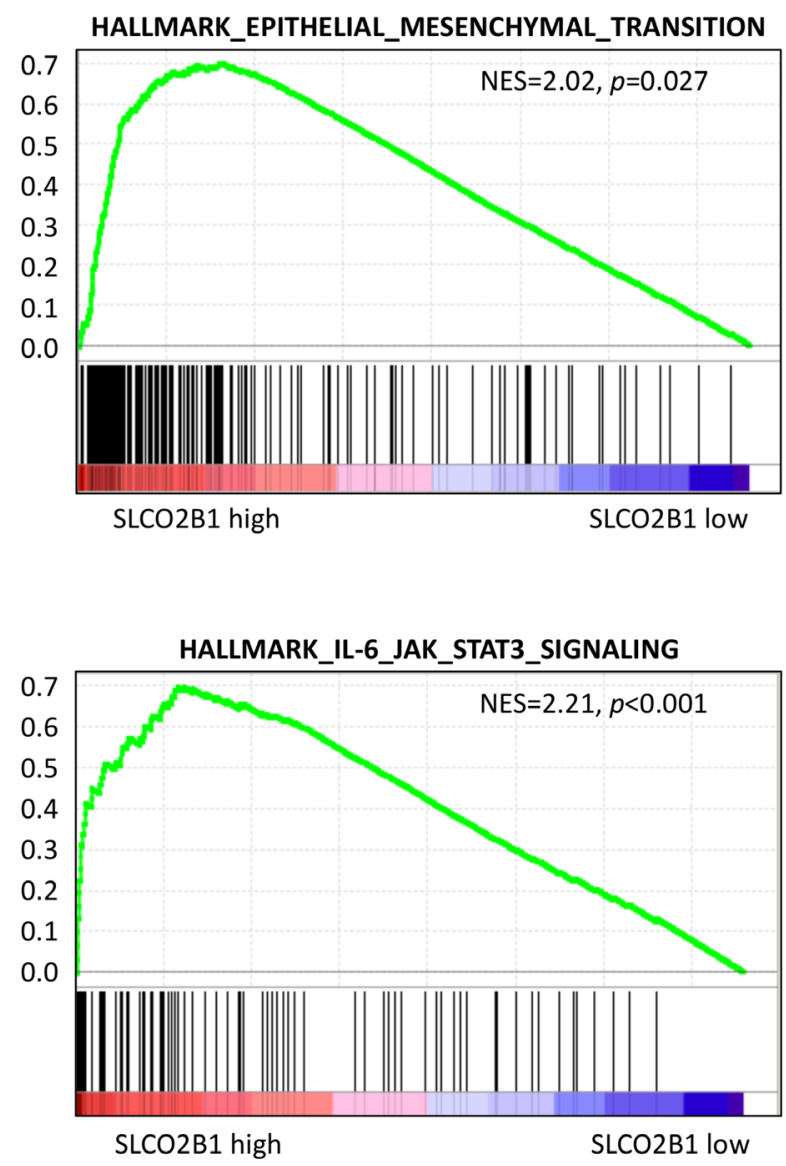

first report showing the clinical relevance of SLCO2B1 expression on the recurrence after RP.

Among a number of SLCO family genes, SLCO2B1 and SLCO1B3 have been implicated in PCa progression, as they both influx androgens such as testosterone and DHEAS which are important precursors to the androgen receptor. A number of studies have shown that SLCO2B1, which encodes OATP2B1, influx endogenous steroids such as DHEAS in PCa. DHEAS uptake has
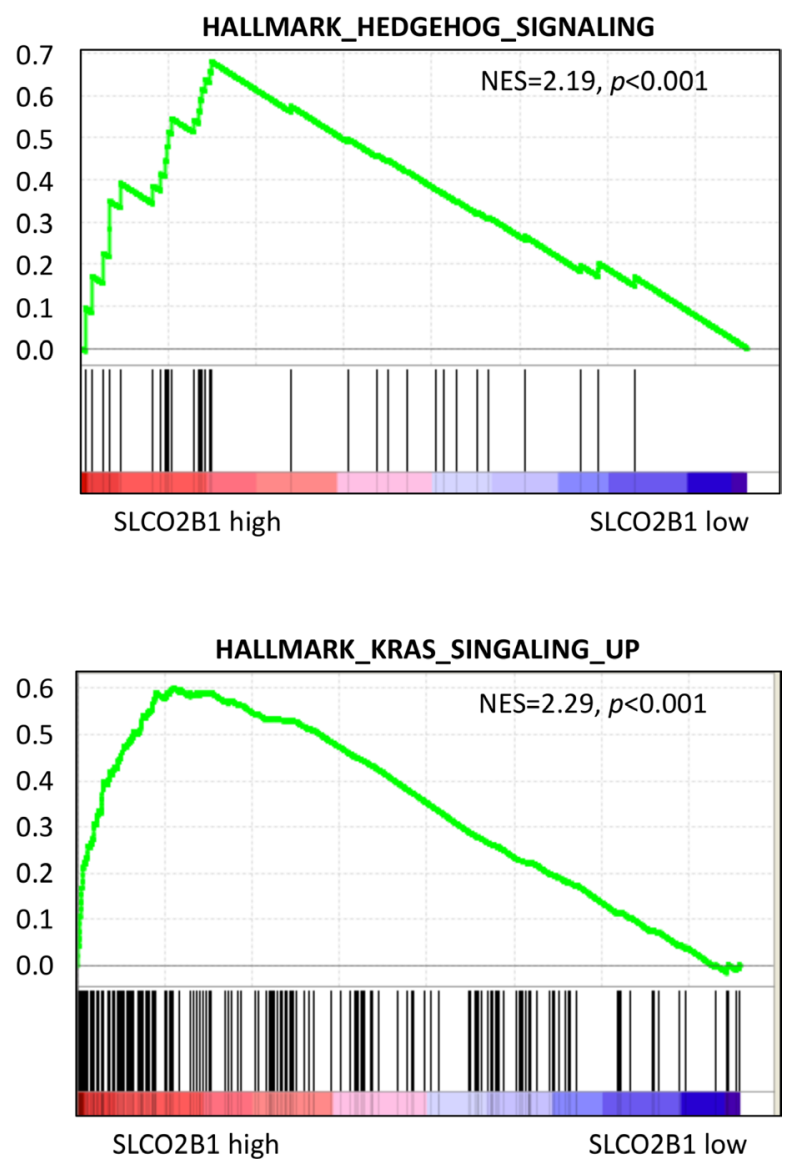

Figure 5: GSEA between SLCO2B1 high and low expression in patients with GS $\geq 8$. 
been shown to be dependent on the expression level of SLCO2B1. Greater expression of SLCO2B1 leads to increased DHEAS transport into cells in PCa cell lines [17], and overexpression of SLCO2B1 increases tumor DHEAS accumulation in a PCa murine model [33]. These findings suggest that SLCO2B1 has an important role in transportation of DHEAS in PCa. Furthermore, genetic variation of SLCO2B1 is associated with resistance to androgen deprivation therapy as well as OS of PCa, whereas there was no difference in recurrence after RP $[17,21,22]$. Among the various members of SLCO gene families, SLCO1B3 which also transports androgen is associated with resistance to hormonal treatment in $\mathrm{PCa}$ $[21,22]$. However, in our results, SLCO1B3 expression was not associated with recurrence after RP, while SLCO2B1 was significantly related and has been shown to be the most important predictor of recurrence after surgery in patients with high GS, compared to other known factors.

To investigate underlying mechanisms, we found that high expression of SLCO2B1 is associated with EMT. It has been shown that the expression of EMT markers is significantly associated with outcomes following RP in localized $\mathrm{PCa}[31,32]$. We previously reported that the expression levels of EMT markers in RP specimens, Twist and Vimentin in particular, was significantly related to recurrence in addition to conventional prognostic parameters [31]. It has also been reported that high expression of Vimentin is a prognostic marker of shorter recurrence-free survival in PCa [32]. In this study, GSEA demonstrated that SLCO2B1 high expression group also enriched gene sets that promote EMT, including the sonic hedgehog pathway, IL-6/JAK/STAT3 pathway and K-Ras signaling pathway related gene sets. Sonic hedgehog pathway has been shown to drive EMT via upregulating $\mathrm{N}$-cadherin and Vimentin in PCa [27]. IL-6/JAK/STAT3 pathway was shown to activate EMT through upregulation of Twist, N-cadherin and Vimentin in some types of cancer [28, 29]. Moreover, EMT has been reported to be promoted by the K-ras signaling pathway [30]. Considering these previous reports and our GSEA results, high expression of SLCO2B1 appears to activate EMT through the upregulation of sonic hedgehog, IL-6/JAK/STAT3 and K-ras signaling pathways. In agreement, DHEA, one of the major metabolites from DHEAS, has been shown to accelerate EMT through E-cadherin suppression and the induction of N-cadherin and Vimentin in PCa [34]. Taken together, it is possible that high expression of SLCO2B1 accelerates the influx of DHEAS, which is then metabolized to the more active DHEA. Accumulated DHEA induces EMT by the activated sonic hedgehog pathway, IL-6/JAK/ STAT3 pathway and K-ras signaling pathway. EMT may promote dissemination and subclinical metastasis prior to RP, leading to recurrence after RP. Although EMT related gene sets were also enriched in SLCO2B1 high group in GS $\leq 7$ patients, these patients were not associated with worse DFS. Considering the result that the expression levels of SLCO2B1 is much higher in GS $\geq 8$ tumors compared to in $\mathrm{GS} \leq 7$ tumors, we speculate that higher expression of SLCO2B1 is required to accelerate this mechanism leading to recurrence in the patients after RP.
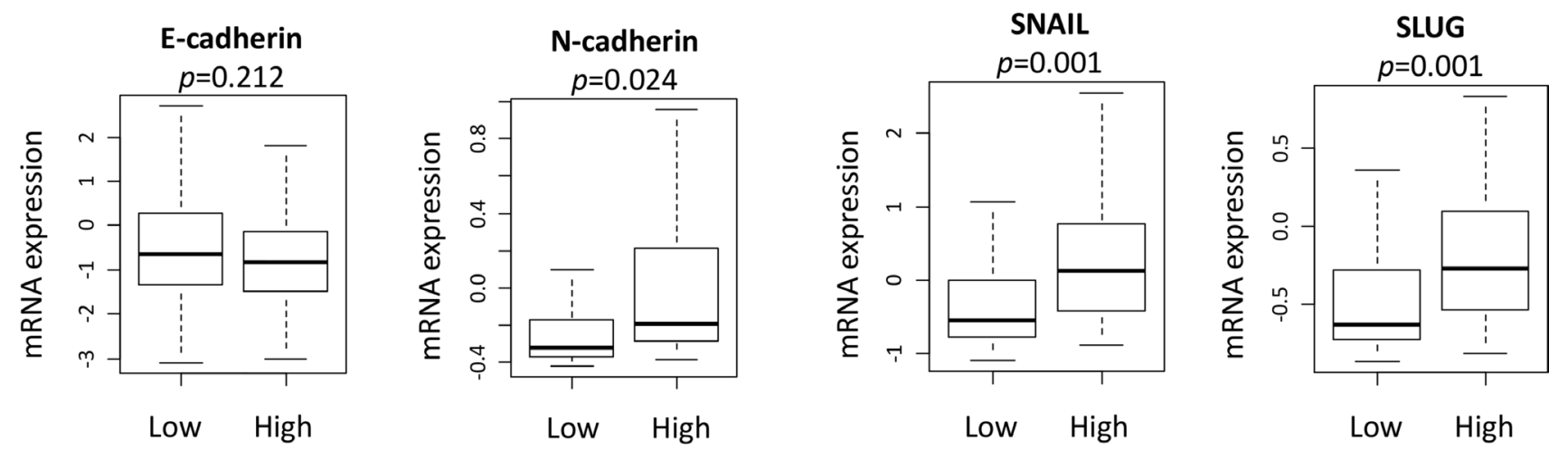

ZEB-1
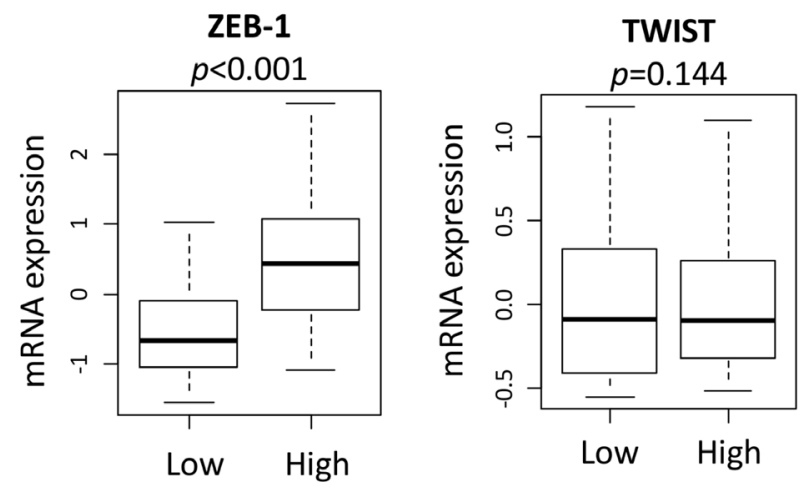

Low High

Low High

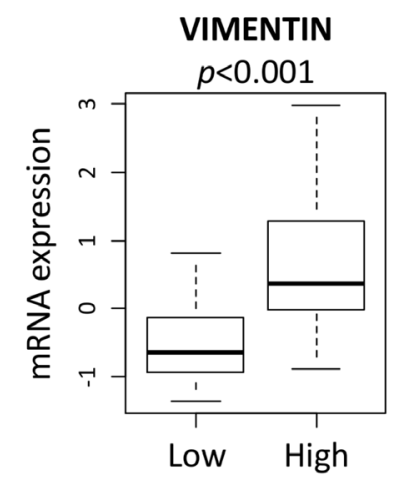

Figure 6: EMT related genes expression comparison between SLCO2B1 high and low in patients with GS $\geq 8$. 
Statins, a class of cholesterol-lowering medications that inhibit 3-hydroxy-3-methl-glutaryl-coenzyme A reductase, are also substrates of SLCO2B1 and have been shown to act as an anticancer drug in various types of cancers [35]. In PCa, clinical studies have shown the controversial results in statin treatment after RP [36-39]. It has been reported that DHEAS uptake is inhibited by statins in PCa cell lines [40], and statin treatment decreased castration resistant progression with lower intra-tumoral androgen levels in murine models [41]. These results suggest that the biological mechanism of statins in PCa may be competitive inhibition of the uptake of DHEAS with SLCO2B1 encoded transporters, OATP2B1. Together with our results, we believe that a randomized clinical trial using high SLCO2B1 expression as a predictive biomarker for statin treatment for GS high $\mathrm{PCa}$ to prevent recurrence after RP is warranted.

Although the present study demonstrates novelty, it has limitations. First, this study was conducted using only one publically available dataset. It will be ideal to compare SLCO2B1 expression level between primary tumor with and without metastasis because EMT is a well-known mechanism in metastatic disease and cancer progression, and validate SLCO2B1 expression level and recurrence using another cohort. However, we were unable to find a dataset which has SLCO2B1 expression level of primary tumor with and without metastasis, as well as SLCO2B1 expression level with patient recurrence-free survival. Secondly, this study is based only on the gene expression of the primary tumor in TCGA cohort and not based on any in vitro and in vivo experiments. In order to determine the role of SLCO gene or to elucidate its molecular mechanism, the experimental approach is needed.

In conclusion, $\mathrm{PCa}$ with high expression of SLCO2B1 in high GS tumors demonstrated worse DFS, which might be due to accelerated EMT. Further studies are needed to determine the clinical application of SLCO2B1 levels.

\section{MATERIALS AND METHODS}

\section{Data acquisition and pre-processing}

There are 498 patients who underwent RP for nonmetastatic disease in the PCa cohort of TCGA. Out of 498, 2 patients with neoadjuvant hormone therapy were excluded from our analysis. Gene expression data and survival data were available in total 494 patients. The gene expression level quantification data (mRNA expression z-score of RNA-seq) were downloaded through cBioPortal and used as previously described [42-44]. Patients were classified into SLCO2B1 high and low groups according to their gene expression level, the cutoff was determined as mean value (0.025). DFS was calculated from time of diagnosis to either biochemical recurrence or radiological tumor recurrence/metastasis.

\section{GSEA of TCGA cohort}

GSEA was performed on TCGA cohort using software provided by the Broad Institute (http://software. broadinstitute.org/gsea/index.jsp), as described before [45-47]. We classified the patients into two groups according to SLCO2B1 expression using same cutoff value (mean: 0.025).

\section{Statistical analysis}

The prognosis differences between SLCO2B1 high and low groups, including OS and DFS, were analyzed using Kaplan-Meier method with log-rank test. Statistical comparisons of the clinicopathological characteristics for significance were performed by the chi-square test or the Fisher exact test, and a Student $t$-test was used to analyze the differences between continuous values. In multivariate analysis, Cox proportional hazards regression method was used in order to identify the most significant independent prognostic factors. In all analysis, a two-sided $p<0.05$ was considered as statistically significant. All statistical analyses were performed using R software (http:///www.rproject.org/) and Bioconductor (http://bioconductor.org/).

\section{Abbreviations}

SLCO: Solute carrier organic anion; PCa: prostate cancer; RP: radical prostatectomy; PSA: prostate specific antigen; OATP: organic anion transport proteins; GS: Gleason score; DHEAS: dehydroepiandrosterone sulfate; TCGA: The Cancer Genome Atlas; DFS: diseasefree survival; OS: overall survival; GSEA: Gene Set Enrichment Analysis; FWER: family wise error rate; EMT: epithelial-mesenchymal transition.

\section{Author contributions}

T. T.: conceptualized and prepared the article. E. K.: performed analysis, prepared the article. L.Y. and N.T.: supervised analysis. K.A.G., K.A.M. and M.F.: edited article. K.T.: provided supervision of analysis and prepared the article.

\section{CONFLICTS OF INTEREST}

There are no potential conflicts of interest to disclose.

\section{FUNDING}

This work was supported by NIH grant R01CA160688 to K.T, and National Cancer Institute (NCI) grant P30CA016056 involving the use of Roswell Park Cancer Institute's Bioinformatics and Biostatistics Shared Resources. 


\section{REFERENCES}

1. Heidenreich A, Bellmunt J, Bolla M, Joniau S, Mason M, Matveev V, Mottet N, Schmid HP, van der Kwast T, Wiegel T, Zattoni F, European Association of U. EAU guidelines on prostate cancer. Part 1: screening, diagnosis, and treatment of clinically localised disease. Eur Urol. 2011; 59:61-71. https://doi.org/10.1016/j.eururo.2010.10.039.

2. Roberts WB, Han M. Clinical significance and treatment of biochemical recurrence after definitive therapy for localized prostate cancer. Surg Oncol. 2009; 18:268-74. https://doi. org/10.1016/j.suronc.2009.02.004.

3. Blute ML, Bergstralh EJ, Iocca A, Scherer B, Zincke H. Use of Gleason score, prostate specific antigen, seminal vesicle and margin status to predict biochemical failure after radical prostatectomy. J Urol. 2001; 165:119-25. https://doi. org/10.1097/00005392-200101000-00030.

4. Nguyen T, Boldt RG, Rodrigues G. Prognostic factors for prostate cancer endpoints following biochemical failure: a review of the literature. Cureus. 2015; 7:e238. https://doi. org/10.7759/cureus.238.

5. Han M, Partin AW, Pound CR, Epstein JI, Walsh PC. Longterm biochemical disease-free and cancer-specific survival following anatomic radical retropubic prostatectomy. The 15-year Johns Hopkins experience. Urol Clin North Am. $2001 ; 28: 555-65$.

6. Hull GW, Rabbani F, Abbas F, Wheeler TM, Kattan MW, Scardino PT. Cancer control with radical prostatectomy alone in 1,000 consecutive patients. J Urol. 2002; 167:528-34.

7. Mian BM, Troncoso P, Okihara K, Bhadkamkar V, Johnston D, Reyes AO, Babaian RJ. Outcome of patients with Gleason score 8 or higher prostate cancer following radical prostatectomy alone. J Urol. 2002; 167:1675-80.

8. Messing EM, Manola J, Sarosdy M, Wilding G, Crawford ED, Trump D. Immediate hormonal therapy compared with observation after radical prostatectomy and pelvic lymphadenectomy in men with node-positive prostate cancer. N Engl J Med. 1999; 341:1781-8. https://doi. org/10.1056/NEJM199912093412401.

9. Messing EM, Manola J, Yao J, Kiernan M, Crawford D, Wilding G, di'SantAgnese PA, Trump D, and Eastern Cooperative Oncology Group study EST 3886. Immediate versus deferred androgen deprivation treatment in patients with node-positive prostate cancer after radical prostatectomy and pelvic lymphadenectomy. Lancet Oncol. 2006; 7:472-9. https://doi.org/10.1016/S1470-2045(06)70700-8.

10. Bianco FJ Jr, Scardino PT, Eastham JA. Radical prostatectomy: long-term cancer control and recovery of sexual and urinary function ("trifecta"). Urology. 2005; 66:83-94. https://doi.org/10.1016/j.urology.2005.06.116.

11. Budaus L, Isbarn H, Eichelberg C, Lughezzani G, Sun M, Perrotte P, Chun FK, Salomon G, Steuber T, Kollermann J, Sauter G, Ahyai SA, Zacharias M, et al. Biochemical recurrence after radical prostatectomy: multiplicative interaction between surgical margin status and pathological stage. J Urol. 2010; 184:1341-6. https://doi.org/10.1016/j. juro.2010.06.018.

12. Mullins JK, Feng Z, Trock BJ, Epstein JI, Walsh PC, Loeb S. The impact of anatomical radical retropubic prostatectomy on cancer control: the 30 -year anniversary. J Urol. 2012; 188:2219-24. https://doi.org/10.1016/j. juro.2012.08.028.

13. Eisenberg MS, Karnes RJ, Kaushik D, Rangel L, Bergstralh EJ, Boorjian SA. Risk stratification of patients with extraprostatic extension and negative lymph nodes at radical prostatectomy: identifying optimal candidates for adjuvant therapy. J Urol. 2013; 190:1735-41. https://doi. org/10.1016/j.juro.2013.05.053.

14. Sissung TM, Baum CE, Kirkland CT, Gao R, Gardner ER, Figg WD. Pharmacogenetics of membrane transporters: an update on current approaches. Mol Biotechnol. 2010; 44:152-67. https://doi.org/10.1007/s12033-009-9220-6.

15. Hagenbuch B, Meier PJ. Organic anion transporting polypeptides of the OATP/ SLC21 family: phylogenetic classification as OATP/ SLCO superfamily, new nomenclature and molecular/functional properties. Pflugers Arch. 2004; 447:653-65. https://doi.org/10.1007/ s00424-003-1168-y.

16. Tamai I, Nezu J, Uchino H, Sai Y, Oku A, Shimane M, Tsuji A. Molecular identification and characterization of novel members of the human organic anion transporter (OATP) family. Biochem Biophys Res Commun. 2000; 273:251-60. https://doi.org/10.1006/bbrc.2000.2922.

17. Wang X, Harshman LC, Xie W, Nakabayashi M, Qu F, Pomerantz MM, Lee GS, Kantoff PW. Association of SLCO2B1 genotypes with time to progression and overall survival in patients receiving androgen-deprivation therapy for prostate cancer. J Clin Oncol. 2016; 34:352-9. https:// doi.org/10.1200/JCO.2015.62.5988.

18. Hagenbuch B, Gui C. Xenobiotic transporters of the human organic anion transporting polypeptides (OATP) family. Xenobiotica. 2008; 38:778-801. https://doi. org/10.1080/00498250801986951.

19. Sharifi N, Hamada A, Sissung T, Danesi R, Venzon D, Baum C, Gulley JL, Price DK, Dahut WL, Figg WD. A polymorphism in a transporter of testosterone is a determinant of androgen independence in prostate cancer. BJU Int. 2008; 102:617-21. https://doi. org/10.1111/j.1464-410X.2008.07629.x.

20. Hamada A, Sissung T, Price DK, Danesi R, Chau CH, Sharifi N, Venzon D, Maeda K, Nagao K, Sparreboom A, Mitsuya H, Dahut WL, Figg WD. Effect of SLCO1B3 haplotype on testosterone transport and clinical outcome in caucasian patients with androgen-independent prostatic cancer. Clin Cancer Res. 2008; 14:3312-8. https://doi. org/10.1158/1078-0432.CCR-07-4118.

21. Wright JL, Kwon EM, Ostrander EA, Montgomery RB, Lin DW, Vessella R, Stanford JL, Mostaghel EA. Expression of 
SLCO transport genes in castration-resistant prostate cancer and impact of genetic variation in SLCO1B3 and SLCO2B1 on prostate cancer outcomes. Cancer Epidemiol Biomarkers Prev. 2011; 20:619-27. https://doi.org/10.1158/1055-9965. EPI-10-1023.

22. Yang M, Xie W, Mostaghel E, Nakabayashi M, Werner L, Sun T, Pomerantz M, Freedman M, Ross R, Regan M, Sharifi N, Figg WD, Balk S, et al. SLCO2B1 and SLCO1B3 may determine time to progression for patients receiving androgen deprivation therapy for prostate cancer. J Clin Oncol. 2011; 29:2565-73. https://doi.org/10.1200/ JCO.2010.31.2405

23. Sooriakumaran P, Srivastava A, Shariat SF, Stricker PD, Ahlering T, Eden CG, Wiklund PN, Sanchez-Salas R, Mottrie A, Lee D, Neal DE, Ghavamian R, Nyirady P, et al. A multinational, multi-institutional study comparing positive surgical margin rates among 22393 open, laparoscopic, and robot-assisted radical prostatectomy patients. Eur Urol. 2014; 66:450-6. https://doi. org/10.1016/j.eururo.2013.11.018.

24. Diaz M, Peabody JO, Kapoor V, Sammon J, Rogers CG, Stricker H, Lane Z, Gupta N, Bhandari M, Menon M. Oncologic outcomes at 10 years following robotic radical prostatectomy. Eur Urol. 2015; 67:1168-76. https://doi. org/10.1016/j.eururo.2014.06.025.

25. Novara G, Ficarra V, Mocellin S, Ahlering TE, Carroll PR, Graefen M, Guazzoni G, Menon M, Patel VR, Shariat SF, Tewari AK, Van Poppel H, Zattoni F, et al. Systematic review and meta-analysis of studies reporting oncologic outcome after robot-assisted radical prostatectomy. Eur Urol. 2012; 62:382-404. https://doi.org/10.1016/j. eururo.2012.05.047.

26. Ondracek RP, Kattan MW, Murekeyisoni C, Yu C, Kauffman EC, Marshall JR, Mohler JL. Validation of the Kattan Nomogram for prostate cancer recurrence after radical prostatectomy. J Natl Compr Canc Netw. 2016; 14:1395-401.

27. Yamamichi F, Shigemura K, Behnsawy HM, Meligy FY, Huang WC, Li X, Yamanaka K, Hanioka K, Miyake H, Tanaka K, Kawabata M, Shirakawa T, Fujisawa M. Sonic hedgehog and androgen signaling in tumor and stromal compartments drives epithelial-mesenchymal transition in prostate cancer. Scand J Urol. 2014; 48:523-32. https://doi. org/10.3109/21681805.2014.898336.

28. Colomiere M, Ward AC, Riley C, Trenerry MK, CameronSmith D, Findlay J, Ackland L, Ahmed N. Cross talk of signals between EGFR and IL-6R through JAK2/STAT3 mediate epithelial-mesenchymal transition in ovarian carcinomas. Br J Cancer. 2009; 100:134-44. https://doi. org/10.1038/sj.bjc.6604794.

29. Kim MS, Lee WS, Jeong J, Kim SJ, Jin W. Induction of metastatic potential by TrkB via activation of IL6/ JAK2/STAT3 and PI3K/AKT signaling in breast cancer. Oncotarget. 2015; 6:40158-71. https://doi.org/10.18632/ oncotarget. 5522 .
30. Zhang K, Myllymaki SM, Gao P, Devarajan R, Kytola V, Nykter M, Wei GH, Manninen A. Oncogenic K-Ras upregulates ITGA6 expression via FOSL1 to induce anoikis resistance and synergizes with alphaV-Class integrins to promote EMT. Oncogene. 2017; 36:5681-94. https://doi. org/10.1038/onc.2017.177.

31. Behnsawy HM, Miyake H, Harada K, Fujisawa M. Expression patterns of epithelial-mesenchymal transition markers in localized prostate cancer: significance in clinicopathological outcomes following radical prostatectomy. BJU Int. 2013; 111:30-7. https://doi. org/10.1111/j.1464-410X.2012.11551.X.

32. Figiel S, Vasseur C, Bruyere F, Rozet F, Maheo K, Fromont G. Clinical significance of epithelial-mesenchymal transition markers in prostate cancer. Hum Pathol. 2017; 61:26-32. https://doi.org/10.1016/j.humpath.2016.10.013.

33. Green SM, Kaipainen A, Bullock K, Zhang A, Lucas JM, Matson C, Banks WA, Mostaghel EA. Role of OATP transporters in steroid uptake by prostate cancer cells in vivo. Prostate Cancer Prostatic Dis. 2017; 20:20-7. https:// doi.org/10.1038/pcan.2016.42.

34. Shin S, Im HJ, Kwon YJ, Ye DJ, Baek HS, Kim D, Choi HK, Chun YJ. Human steroid sulfatase induces Wnt/betacatenin signaling and epithelial-mesenchymal transition by upregulating Twist1 and HIF-1alpha in human prostate and cervical cancer cells. Oncotarget. 2017; 8:61604-17. https:// doi.org/10.18632/oncotarget.18645.

35. Nielsen SF, Nordestgaard BG, Bojesen SE. Statin use and reduced cancer-related mortality. N Engl J Med. 2012; 367:1792-802. https://doi.org/10.1056/NEJMoa1201735.

36. Park HS, Schoenfeld JD, Mailhot RB, Shive M, Hartman RI, Ogembo R, Mucci LA. Statins and prostate cancer recurrence following radical prostatectomy or radiotherapy: a systematic review and meta-analysis. Ann Oncol. 2013; 24:1427-34. https://doi.org/10.1093/annonc/mdt077.

37. Scosyrev E, Tobis S, Donsky H, Wu G, Joseph J, Rashid H, Messing E. Statin use and the risk of biochemical recurrence of prostate cancer after definitive local therapy: a metaanalysis of eight cohort studies. BJU Int. 2013; 111:E71-7. https://doi.org/10.1111/j.1464-410X.2012.11527.x.

38. Allott EH, Howard LE, Cooperberg MR, Kane CJ, Aronson WJ, Terris MK, Amling CL, Freedland SJ. Serum lipid profile and risk of prostate cancer recurrence: Results from the SEARCH database. Cancer Epidemiol Biomarkers Prev. 2014; 23:2349-56. https://doi.org/10.1158/1055-9965. EPI-14-0458.

39. Tan P, Wei S, Yang L, Tang Z, Cao D, Liu L, Lei J, Fan Y, Gao L, Wei Q. The effect of statins on prostate cancer recurrence and mortality after definitive therapy: a systematic review and meta-analysis. Sci Rep. 2016; 6:29106. https://doi.org/10.1038/srep29106.

40. Harshman LC, Wang X, Nakabayashi M, Xie W, Valenca L, Werner L, Yu Y, Kantoff AM, Sweeney CJ, Mucci LA, Pomerantz M, Lee GS, Kantoff PW. Statin use at th e time of initiation of androgen deprivation therapy and time to 
progression in patients with hormone-sensitive prostate cancer. JAMA Oncol. 2015; 1:495-504. https://doi. org/10.1001/jamaoncol.2015.0829.

41. Gordon JA, Midha A, Szeitz A, Ghaffari M, Adomat HH, Guo Y, Klassen TL, Guns ES, Wasan KM, Cox ME. Oral simvastatin administration delays castration-resistant progression and reduces intratumoral steroidogenesis of LNCaP prostate cancer xenografts. Prostate Cancer Prostatic Dis. 2016; 19:21-7. https://doi.org/10.1038/ pcan.2015.37.

42. Center MSKC. cBioPortal. http://www.cbioportal.org/.

43. Kim SY, Kawaguchi T, Yan L, Young J, Qi Q, Takabe K. Clinical relevance of microRNA expressions in breast cancer validated using the Cancer Genome Atlas (TCGA). Ann Surg Oncol. 2017; 24:2943-49. https://doi. org/10.1245/s10434-017-5984-2.

44. Ramanathan R, Olex AL, Dozmorov M, Bear HD, Fernandez LJ, Takabe K. Angiopoietin pathway gene expression associated with poor breast cancer survival. Breast Cancer Res Treat. 2017; 162:191-8. https://doi. org/10.1007/s10549-017-4102-2.

45. Kawaguchi T, Yan L, Qi Q, Peng X, Gabriel EM, Young J, Liu S, Takabe K. Overexpression of suppressive microRNA, miR-30a and miR-200c are associated with improved survival of breast cancer patients. Sci Rep. 2017; 7:15945. https://doi.org/10.1038/s41598-017-16112-y.

46. Young J, Kawaguchi T, Yan L, Qi Q, Liu S, Takabe K. Tamoxifen sensitivity-related microRNA-342 is a useful biomarker for breast cancer survival. Oncotarget. 2017; 8:99978-89. https://doi.org/10.18632/oncotarget.21577.

47. Katsuta E, Yan L, Nagahashi M, Raza A, Sturgill JL, Lyon DE, Rashid OM, Hait NC, Takabe K. Doxorubicin effect is enhanced by sphingosine-1-phosphate signaling antagonist in breast cancer. J Surg Res. 2017; 219:202-13. https://doi.org/10.1016/j.jss.2017.05.101. 